
\title{
R Research Soure \\ The bone flare phenomenon during the treatment of bone metastases of lung adenocarcinoma
}

Jianhua Shi

Linyi Cancer Hospital

Guimin Chen

Linyi cancer Hospital

Tianhui Xu

Linyi Cancer Hospital

\section{Xiuxiu Wang}

Linyi Cancer hospital

Junxia Ruan

Linyi People's Hospital

Shuoxin Liu

Linyi Cancer Hospital

Shengqiang Sun

Linyi Cancer Hospital

\section{Yupeng Li}

Linyi Cancer Hospital

Jinping Zhang

Linyi Cancer Hospital

Chunling Ma ( $\triangle$ machl2003@163.com )

Shandong Medical College

\section{Research}

Keywords: Bone Flare, Lung Adenocarcinoma, Bone Metastasis, Alkaline Phosphatase, Serum Calcium

Posted Date: June 4th, 2021

DOI: https://doi.org/10.21203/rs.3.rs-518758/v1

License: (1) This work is licensed under a Creative Commons Attribution 4.0 International License.

Read Full License 
The bone flare phenomenon during the treatment of bone metastases of lung adenocarcinoma

Jian Shi ${ }^{1}$, Guimin chen ${ }^{1}$, Tianhui $\mathrm{Xu}^{1}$, Xiuxiu Wang${ }^{1}$, Junxia Ruan ${ }^{2}$, Shuoxin Liu ${ }^{1}$, Shengqiang Sun ${ }^{1}$, Yupeng $\mathrm{Li}^{1}$, Jinping Zhang ${ }^{1}$, Chunling $\mathrm{Ma}^{3}$

${ }^{1}$ Department of Second Internal Medicine, Linyi Cancer Hospital, Linyi, Shandong 276000, P.R. of China; ${ }^{2}$ Birth Defect Study Laboratory, Linyi Maternal and Child Healthcare Hospital, Linyi, Shandong 276000, P.R. of China; ${ }^{3}$ Experimental Center of Molecular Biology, Shandong Medical College, Linyi, Shandong 276000, P.R. of China.

Correspondence to: Chunling Ma, Experimental Center of Molecular Biology, Shandong Medical College, Linyi, Shandong 276000, P.R. China, E-mail:machl2003@163.com

\begin{abstract}
:
Background: The bone flare phenomenon, defined as an increase in bone lesion activity, is a kind of benign bone change in response to ongoing tumor treatment. The purpose of this study was to investigate the time, incidence, and clinical significance of bone flare in lung adenocarcinoma patients with bone metastases, as well as to observe the levels of serum alkaline phosphatase $(A L P)$ and serum calcium $\left(\mathrm{Ca}^{2+}\right)$ in patients with bone flare phenomenon.
\end{abstract}

Methods: Fifty-seven patients with advanced lung adenocarcinoma who participated in the anti-tumor clinical trials from December 2017 to December 2019 in Linyi Cancer Hospital were included in the study. The CT (computed tomography, CT) images, serum $A L P$ and $\mathrm{Ca}^{2+}$ from all patients were analyzed retrospectively.

Results: Among a total of 57 patients, 28 were male, and 29 were female. The median age was 62 years (33-75 years), and 30 of them had bone metastases at baseline. Forty-six EGFR negative patients received platinum-based dual-drug chemotherapy or bevacizumab or combined with PD-1 antibody inhibitors, and 11 EGFR positive patients received targeted EGFR inhibitors. The bone flare was detected in 7 out of 30 patients with bone metastases at baseline ( 5 patients in the combined chemotherapy group and two patients in the targeted treatment group). The incidence of bone flare was $23.33 \%$ (7 / 30). The median time was 45 days after treatment (from 41 days to 120 days), and most of them occurred in the early stages of the treatment.

Of the 30 patients with bone metastases at the beginning of treatment, twenty had $A L P$ and $\mathrm{Ca}^{2+}$ levels observed over five months, seven of them with bone flare, 13 with benefit (partial response,PR/stable disease,SD), and 5 with progression (progressive disease, PD). Analysis of 
$A L P$ from patients with different curative effects showed significant changes compared with the median percentage of baseline levels. The bone flare group and the beneficial group showed a decreasing trend. The bone flare group showed a more significant decreasing trend, $A L P$ levels in the progressive group increased continuously. The percentage change of serum $\mathrm{Ca}^{2+}$ during the treatment was not noticeable.

Conclusion: The bone flare phenomenon was observed among patients with lung adenocarcinoma with bone metastasis. Most of them occur in the early stage of treatment. Combined with clinical evidence such as $A L P$, physicians and radiologists should avoid a misinterpretation of bone flare as the progression of metastatic bone cancer and stopping the effective treatment too early.

Keywords: Bone Flare; Lung Adenocarcinoma; Bone Metastasis; Alkaline Phosphatase; Serum Calcium

\section{Background}

Lung cancer is one of the most common malignant tumors in the world ${ }^{[1]}$. According to the histological classification, lung cancer was classified as small-cell carcinoma (SCLC, about $15 \%$ of lung cancers) and non-small-cell lung carcinoma (NSCLC, about $85 \%$ of lung cancers) ${ }^{[2]}$. Among them, lung adenocarcinoma was the most common subtype in NSCLC, accounting for about $40 \%$ of all lung cancers ${ }^{[3]}$, and the incidence is increasing ${ }^{[4]}$.

Some studies have shown that the incidence of bone metastasis in NSCLC patients is about $30-40 \%{ }^{[5,6]}$, of whom $60 \%$ of the patients had bone metastasis at the time of diagnosis $^{[7]}$, and most of them were osteolytic, and a few were osteogenic or mixed ${ }^{[8]}$. With the development of cancer science, chemotherapy, targeted therapy, and immunotherapy have greatly improved the efficacy and survival time of advanced lung adenocarcinoma. However, in clinical practice, we have found that some patients in the early stage of treatment, primary lesions or other metastatic lesions all suggest a reduction or stabilization, but the density or number of metastatic bone lesions increased. Some patients have changed the treatment regimen according to their disease 
progression, but the lesions in other sites increased rapidly; some patients continued to be treated according to the original regimen to keep the tumor under control.

Upon inquiry, it was found that this treatment for Central Plain Foci or other metastatic foci was well controlled and resulted in a "progression of bone metastases," known as "bone flare"[9]. Bone flare is a manifestation of systemic therapy for malignant tumors, not a progression of metastatic bone tumors. As treatment continues, bone lesions remain stable or improve. Also, serum ALP and $\mathrm{Ca}^{2+}$ as markers of bone metabolism are closely related to bone metastasis of lung cancer ${ }^{[10,11]}$. Based on this, we retrospectively analyzed 57 patients with newly diagnosed advanced lung adenocarcinoma from 5 clinical trials. In the 7 cases, BMD (bone material density, $\mathrm{BMD}$ ) or BMD number increased while other lesions were relieved or stable during anti-tumor therapy. We compared the changes in bone window by CT imaging, serum ALP and $\mathrm{Ca}^{2+}$ levels before and after treatment to observe the bone lesions in lung adenocarcinoma patients with bone metastasis during anti-tumor therapy, to distinguish the progression of bone metastasis or bone flare, and to look for the markers related to bone metabolism.

\section{Materials and methods}

\subsection{Subjects}

The patients included in the study were treated at the Linyi Cancer Hospital, Linyi. Inclusion criteria were (1) patients aged from 18 to 75 years who had been diagnosed with stage IIIB / IV lung adenocarcinoma; (2) patients with at least one measurable lesion evaluated according to Recist1.1; (3) Patients had been assessed with ECOG PS (Eastern Cooperative Oncology Group Performance Status, ECOG PS) 0-1; (4) The expected survival time is greater than or equal to 3 months; (5) All patients have signed informed consent for treatment, which has been approved by the hospital ethics committee.

From December 2017 to December 2019, 57 patients with advanced lung adenocarcinoma were enrolled in five clinical trials. Among all of the patients, 28 
were male, and 29 were female. The age of patients ranged from 33 to 75 years, and the median age was 62 years. Six of these patients had stage IIIB disease, and 51 of whom had IV disease. Forty-six patients were EGFR negative, and 11 were EGFR positive. Also, 30 patients had bone metastases at baseline.

\subsection{Treatment}

1.2.1Combination chemotherapy: Among 46 EGFR negative patients, 35 patients received the regimen of bevacizumab plus Paclitaxel plus Carboplatin, 5 patients received PD-1 inhibitor plus pemetrexed plus Carboplatin, and 6 patients received pemetrexed plus Carboplatin chemotherapy.

1.2.2 Targeted therapy group: 11 EGFR positive patients received the first-generation EGFR-TKI targeted therapy.

\subsection{Methods}

\subsubsection{Imaging examination:}

All CT scans were performed with an enhanced 64-slice spiral scanner to define the extent of bone lesions.Scanning parameters: Tube Voltage: $120 \mathrm{KV}$; tube current:150mAS; layer thickness: $5 \mathrm{~mm}$; layer distance:5mm; the contrast medium was iohexol; the dosage was from 1.5 to $2.0 \mathrm{ml} / \mathrm{kg}$. The injection rate was from 2.5 to $3.5 \mathrm{ml} / \mathrm{h}$ through the median elbow vein with a high-pressure syringe. Follow-up was performed every six weeks, and enhanced CT was used to evaluate the therapeutic effect. The efficacy was assessed based on RECIST1.1 criteria.

\subsubsection{Laboratory examination:}

Serum ALP and $\mathrm{Ca}^{2+}$ levels were measured at baseline and before treatment in the next cycle. Three milliliters of fasting venous blood was collected and detected by Beckman Coulter AU5800 automatic biochemical analyzer. The reference value of ALP was from 45 to $125 \mathrm{U} / \mathrm{L}$; the reference value of $\mathrm{Ca}^{2+}$ level ranged from 2.03 to $2.60 \mathrm{mmol} / \mathrm{L}$.

\subsubsection{Statistical analysis}

All experiments are presented as the mean $\pm \mathrm{S}$. Student's $t$-test was used for statistical 
evaluations of two group comparisons. One-way analysis of variance (ANOVA) for more than two groups was performed for statistical analysis.

\section{Results}

Thirty patients of the 57 subjects had bone metastases at baseline, 7 had bone flare after treatment ( 5 cases in the combined group and 2 cases in the targeted group), the incidence rate was $23.33 \%$ (Table 1). The time of bone flare occurrence was 41 to 125 days after treatment, and the median time was 45 days. Five cases $(71.43 \%)$ had bone flare within 50 days of treatment (Table 2). All patients continued the initial treatment after the occurrence of bone flare. CT scans showed that the soft tissue lesions were relieved or stable. The patients felt better during the treatment and did not display symptoms associated with pain in the bone.

Among 30 patients with bone metastases at baseline, 25 cases had serum ALP and $\mathrm{Ca}^{2+}$ values observed over five months, of which 7 cases had bone flare, 13 cases were of benefit (PR/SD), 5 cases were of progression (PD). In 25 patients, no significant change was found in the percentage of serum $\mathrm{Ca}^{2+}$ compared with the median values of that in the baseline. Regardless of the effect of treatment, while the serum ALP showed significant change (see Figure 1), the bone flare group and the benefit group showed a downward trend, and the bone flare group showed a more significant downward trend, while the serum ALP level in the progressive group showed a continuous upward trend. The ALP value of 7 patients with bone flare was followed up. The ALP value of 4 patients with bone flare increased temporarily, then decreased slowly, and the ALP value of the other three patients continued to decline (see Figure 2).

\section{Case report}

\subsection{The ${ }^{\#} 1$ patient of typical bone flare}

A 53-year-old man went for a physical examination and a right lung nodule was found in July 2017. Thoracoscopic radical resection of right upper lobe lung cancer was performed on August 23, 2017, pT1N0M0. He was diagnosed with stage IA lung cancer, and he did not receive special anti-tumor therapy after operation. On February 
19,2018 , the patient was admitted for the second time due to left shoulder pain four months after the right lung cancer operation. Examinations were performed by enhanced CT and bone scan, and showed lymph node, liver, and bone metastasis (cTxN2M1 IV stage), ALP: $142 \mathrm{U} / \mathrm{L}, \mathrm{Ca}^{2+}: 2.53 \mathrm{mmol} / \mathrm{L}$. On February 22, 2018, the patient was enrolled to a clinical study by using PD-1 inhibitor in combination with systemic chemotherapy for newly treated lung adenocarcinoma. He was treated with PD-1 inhibitor in combination with pemetrexed disodium plus carboplatin regimen in our hospital. Symptoms of left shoulder pain decreased after 3 weeks in 1 cycle, ALP: $144 \mathrm{U} / \mathrm{L}, \mathrm{Ca}^{2+}: 2.52 \mathrm{mmol} / \mathrm{L}$; After 6 weeks in 2 cycles, the left shoulder pain disappeared, ALP: $145 \mathrm{U} / \mathrm{L}, \mathrm{Ca}^{2+}: 2.55 \mathrm{mmol} / \mathrm{L}$. On April 04, 2018, lung lesions (Figure 3B) and liver metastases (Figure 4B) were significantly reduced and new osteogenic changes in the Thoracic 5 Vertebra (Figure 5B) were observed by CT examination.

After analysis, we found that both primary lesions and intrahepatic metastases of patients were reduced, had no new symptoms of bone pain, no abnormal changes in bone metabolism. Bone destruction in the Thoracic 5 vertebral body may be a response to osteogenic healing, and treatment with the original regimen had been continued for 6 cycles, and the evaluation was PR.

On July 13, 2018, ALP: $98 \mathrm{U} / \mathrm{L}, \mathrm{Ca}^{2+}: 2.30 \mathrm{mmol} / \mathrm{L}$, CT showed that both lung lesions and liver metastases were significantly reduced (Figure 3D, 4D). The changes of thoracic 5 vertebral body were the same as before (Figure 5D). After that, he was treated with a single drug, pemetrexed, the ALP: $68 \mathrm{U} / \mathrm{L} \mathrm{Ca}^{2+}: 2.48 \mathrm{mmol} / \mathrm{L}$ were from reexamined on September 24, 2019. CT showed that the lesions were stable and no further progression were found in bone metastases.

\subsection{The \# 2 patient of typical bone flare}

The patient was a middle-aged woman who presented with a cough in November 2018. Chest CT revealed a mass lesion in the right upper lobe of the lung. CT-guided percutaneous biopsy of the lung mass from December 18, 2018 showed adenocarcinoma of the lung carrying EGFR21-L858R mutation. She was diagnosed with stage IV right lung adenocarcinoma CT2N2M1(bone). The results of serological 
testing on January 4, 2019 were ALP: $164 \mathrm{U} / \mathrm{L}, \mathrm{Ca}^{2+}: 2.14 \mathrm{mmol} / \mathrm{L}$.Beginning from January 9,2019, the first generation of EGFR-TK1 targeted drug was given orally. The cough symptoms were alleviated after taking EGFR-TK1. After 6 weeks of follow-up, CT showed that the primary lung lesion was smaller than before (Figure $6 \mathrm{~B}$ ), and the osteogenic bone metastasis was slightly larger (Figure 7B) with serum ALP $134 \mathrm{U} / \mathrm{L}, \mathrm{Ca}^{2+}: 2.02 \mathrm{mmol} / \mathrm{L}$. Three months later, the patient had no cough symptoms and no pain. CT scan showed stable lung lesions (Figure 6C), increased density of osteogenic bone metastases (Figure 7C), ALP: $69 \mathrm{U} / \mathrm{L}$, $\mathrm{Ca}^{2+}: 1.96 \mathrm{mmol} / \mathrm{L}$. After analysis of clinical manifestation, imaging and laboratory examination, considering the osteogenic healing reaction, she continued to receive the EGFR-TK1 targeted therapy, and the curative effect was evaluated continuously.

\section{Discussion}

The bone flare phenomenon was first reported in 1976 and is usually seen in advanced malignant tumor patients that respond well to systemic anti-cancer therapy, such as breast cancer and prostate cancer patients. The incidence and time of bone flare are different in each study due to different types of diseases and treatment schemes. With the development of targeted therapy and immunotherapy, the response to NSCLC treatment is improved, which indicates the incidence of bone flare. In 2009, Lind JS, et al. first reported osteoblast flare phenomenon in 3 NSCLC patients treated in Erlotinib $^{[15]}$. Heng-sheng Chao et al. reported that bone flare occurred in 7 of 33 NSCLC patients with bone metastatic treated with gefitinib, with a median time of 34 days ${ }^{[9]}$. Yelena Krupitskaya, et al. also reported four patients with NSCLC who experienced a solitary deterioration of bone metastases during bevacizumab combined with standard chemotherapy. Subsequent scans confirmed that the deterioration was transient and consistent with flare reaction ${ }^{[16]}$. In summary, bone flare is not an adverse reaction, but a phenomenon of osteoblast activation during bone repair ${ }^{[17-19]}$. It is characterized by rapid bone tissue repair around bone lesions and is an effective marker for systemic treatment of malignant tumors, which is not a tumor bone metastases progression $^{[20]}$. 
At present, the causes of the bone flare phenomenon are not very clear, which may be related to the changes in cancer cells and bone microenvironment in bone metastases lesions after effective treatment. In normal bone tissues, there are two main types of cells responsible for bone remodeling, osteoclasts (absorb bone tissue) and osteoblasts (form bone tissue). Bone metastases usually involve both osteoblasts and osteoclasts, and their interaction predisposes the tumor to osteogenesis or osteolysis. Bone metastases from lung cancer are usually bone resorption by osteoclasts, i.e., osteolytic changes. Bone metastasis is a multi-step process, many factors and signal pathways interact between cancer cells and the microenvironment, which leads to an imbalance of normal bone remodeling and promotes the development of bone metastasis. Research data show that cancer cells migrate to the bone and release soluble mediators, activate osteoclasts and osteoblasts, and the cytokines released by osteoclasts further promote cancer cells to secrete osteolytic mediators, forming a vicious circle, and then there's osteolytic change ${ }^{[21]}$. Other studies have reported that many factors can promote the growth and differentiation of osteoblasts ${ }^{[16]}$.

We hypothesized that the bone flare phenomenon might be caused by the reduction of tumor load in bone metastases, the reduction of osteolytic mediators released by cancer cells, and the reduction of osteoclast activity. On the other hand, when anti-tumor therapy is effective, the changes of tumor-derived factors caused by changes in the tumor microenvironment can promote the proliferation and differentiation of osteoblasts and lead to increased uptake of bone imaging, thus leading to rapid bone repair and healing, and the bone flares formed. If the bone lesions continue to respond to treatment, the increase in uptake will eventually decrease ${ }^{[22]}$. Previous studies have found that bone flares typically occur in the early months of treatment ${ }^{[23]}$, usually from 2 weeks to 3 months after treatment, but rarely 6 months after treatment, and it was included as part of the MD Anderson Cancer Center's bone metastasis treatment remission criteria. In this study, bone flare phenomenon occurred in 5 of 7 patients $(71.43 \%)$ about 6 weeks after treatment. The other 2 cases occurred within 4 months after treatment, which was consistent with the literature. Multi-slice spiral CT (MSCT) is one of the most important imaging 
methods for the diagnosis and evaluation of malignant tumors, which is very important to distinguish between the phenomenon of bone flare and bone metastasis. In the past, bone scintigraphy and bone Single-photon emission computed tomography have been reported in detail in the clinical study of bone metastases, but Multi-slice spiral CT has rarely been reported in the diagnosis of bone flare.

In 2004, the University of Texas developed the bone-specific Efficacy Standard (MDA standard), which updates the UICC and World Health Organization (WHO) bone efficacy evaluation standards to include CT and MRI for radiologic evaluation. CT Bone window examination can provide anatomical information, and can clearly show the shape, contour, density, and other abnormalities of bone lesions, high resolution, sensitivity can reach $77 \%{ }^{[24]}$. As mentioned in the MDA standard, the definition of PR should pay attention to the phenomenon of osteoblast bone flare ${ }^{[25]}$, in the case of other lesions PR, osteosclerosis or osteolytic lesions during treatment of marginal sclerosis, it does not represent tumor progression but healing of bone lesions that were not evident prior to treatment ${ }^{[26]}$. The 7 patients with bone flare in our study were all patients with bone metastasis at baseline. After treatment, Osteogenic sclerosis reaction occurred in the osteolytic destruction lesions.

Bone metabolic biomarkers can reflect the rate of bone resorption and the speed of bone formation during bone metastasis and indicate the degree of bone destruction and repair ${ }^{[27]}$. As a marker of bone metabolism closely related to bone metastasis of lung cancer, serum alkaline phosphatase and serum calcium $\left(\mathrm{Ca}^{2+}\right)$ are often used to assist the diagnosis and follow-up of bone metastasis. ALP is an enzyme expressed on the surface of osteoblasts, which can affect the activity of osteoblasts. In osteolytic bone metastasis, the activity of ALP increased after local bone formation reaction in order to compensate for the major osteoclast lesions, while in osteogenic bone metastasis, the activity of ALP increased after local stimulation by osteoblasts ${ }^{[29]}$. Huang et al. (meta-analysis) found that ALP level was higher in patients with bone metastasis of lung cancer than in patients without bone metastasis, suggesting that serum ALP level is an indicator for bone metastasis in lung cancer ${ }^{[3]}$. Karhade-1 et al. also found that ALP was significantly higher in patients with multiple column 
metastases than in patients without column metastases. Effective bone metastases therapy can reduce bone-related adverse events, and it can be reflected through the reduction of biomarkers of bone metabolism, including the ALP.

In 25 patients with bone metastases at baseline and have serum ALP values during treatment, the ALP of clinical benefit group (including bone flare patients) showed a decreasing trend compared with the median percentage at baseline, and the decrease trend was more significant in bone flare group. The ALP values of the patients in the progressive group showed an increasing trend, which also suggested that ALP could be used as a marker of effective treatment for patients with bone metastasis of lung cancer, which is consistent with previous reports. In this study, osteogenic sclerosis in 7 patients with bone flare was benign rather than progressive. In addition, ALP levels in the flare group decreased overall, but in 4 patients there was a transient increase in the flare, followed by a slow decline, which may be due to osteolytic destruction after effective treatment, the activity of osteoblasts increased in the early stage, which led to a transient increase of ALP level. With the continuous reaction and mineralization, the activity of osteoblasts decreased gradually, and the level of ALP also decreased gradually.

Elevated serum $\mathrm{Ca}^{2+}$ levels has been found in a wide range of soluble osseous bone metastases. On one hand, tumor cells produce protein decomposing enzyme can cause bone matrix dissolution, destroy ${ }^{[21]}$; on the other hand, the cancer bone metastases can produce a variety of promote the activity of bone absorption and bone calcium release factor ${ }^{[16]}$, eventually dissolved bone calcium release exceeds the ability of the kidney and intestine removing calcium, which may lead to increased calcium in the blood. Bone damage caused by lung cancer metastasis is usually osteolytic, leading to an increase in serum $\mathrm{Ca}^{2+}$ when bone metastasis occurs ${ }^{[27]}$. However, in this study, during the whole treatment period, 25 patients with bone metastasis at baseline and complete serum $\mathrm{Ca}^{2+}$ data were not observed with significant changes in the percentage of $\mathrm{Ca}^{2+}$ compared with the median baseline in different curative effects, and the number of cases should be expanded for further observation. 


\section{Conclusion:}

In conclusion, bone flare can be considered as a sign of efficiency in systemic therapy for advanced malignant tumors. With the improvement of lung cancer therapies and the development of imaging diagnosis, the bone flare phenomenon is now observed more commonly in patients with bone metastasis of lung cancer. Therefore, clinicians and radiologists should pay attention to bone flare phenomenon and avoid early termination of effective treatment due to misdiagnosis as progression of cancer. Future research should focus on the causes and mechanisms of this phenomenon in order to better guide clinical work.

\section{List of Abbreviations:}

Alkaline phosphatase, ALP; Calcium; $\mathrm{Ca}^{2+}$; Computed tomography, CT; Non-small-cell lung carcinoma, NSCLC; Bone material density, BMD; Eastern Cooperative Oncology Group Performance Status, ECOG PS; Epidermal growth factor receptor, EGFR; Epidermal growth factor receptor-tyrosine kinase inhibitor, EGFR-TKI ; Programmed death receptor-1, PD-1; Multi-slice spiral CT, MSCT.

\section{Declarations:}

Ethics approval and consent to participate: This study was approved by the Ethics Committee of the Linyi Cancer Hospital and all the patients in the study signed informed consent forms.

Consent for publication: All authors agreed to publish the draft and transfer the copyright to the publisher.

Availability of data and material: Not applicable

Competing interests: The authors declare no competing financial interests.

Funding: Natural Science Foundation of Shandong Province (ZR2017MH059 to CM).

Authors' contributions:JS and GC carried out studies, and drafted the manuscript in Chinese. JS, TX SL and SS participated in the design of the study and performed the statistical analysis. JS, YL, JZ and CM conceived of the study, and participated in its design and coordination. CM and JR helped to draft the manuscript in English. All authors read and approved the final manuscript. 
Acknowledgments: The authors would like to thank for our colleagues, SW and CG, in the imaging department, providing help with patient imaging studies, and ZM and JS for statistical analysis.

\section{Reference:}

[1]. Jemal A, Bray F, Center MM, Ferlay J, Ward E, Forman D. Global cancer statistics. CA Cancer J Clin 2011;61:69-90.

[2]. Sher, T., Dy, G. K. \& Adjei, A. A. Small cell lung cancer. Mayo. Clin. Proc. 83,355-367 (2008).

[3]. Rosen, L. S. et al. Long-term efficacy and safety of zoledronic acid in the treatment of skeletal metastases in patients with non-small cell lung carcinoma and other solid tumors. A randomised, Phase III, double blind, placebo-controlled trial. Cancer. 100, 2613-2621(2004).

[4]. Price, N., Belani, C. P. \& Jain, V. K. Biphosphonates to prevent skeletal morbidity in patients with lung cancer with bone metastases.Clin Lung Cancer. 5, 267-269 (2004)

[5]. Noguchi, M., Morikawa, A. \& Kawasaki, M. et al. Small adenocarcinoma of the lung. Histologic characteristics and prognosis. Cancer 75, 2844-2852 (1995).

[6]. Zappa, C. \& Mousa, S. A. Non-small cell lung cancer: current treatment and future advances. Transl Lung Cancer Res. 2016 Jun;5(3):288-300.

[7]. Herbst R.S.,HeymachJ.V.,LippmanS.M.Lungcancer.N.Engl.J.Med.2008;359:1367-1380.

[8]. Hashisako M, Wakamatsu K, Ikegame S, Kumazoe H, Nagata N, KajikiA. Flare phenolmenon following gefitinib treatment of lung adenocarcinoma with bone metastasis.Tohoku J Exp Med.2012 Oct;228(2):163-8.

[9]. Chao HS, Chang CP, Chiu CH, Chu LS, Chen YM, Tsai CM.Bone Scan Flare Phenomenon in Non-Small-Cell Lung Cancer Patients Treated With Gefitinib. Clin Nucl Med.2009 Jun;34(6):346-9.

[10]. Du W X, Duan S F , Chen J J , et al. Serum bone-specific alkaline phosphatase as a biomarker for osseous metastases in patients with malignant carcinomas: A systematic review and meta-analysis[J]. Journal of Cancer Research \& Therapeutics, 2014, 10 suppl:C140-3.

[11]. Bone. 1987;8 Suppl 1:S17-22. Bone metastases in breast cancer, prostate cancer and myeloma.

[12]. Alexander JL, Gilliespie PJ, Edelstyn GA. Serial bone scanning using technetium 99m diphosphonate in patients undergoing cyclical combined chemotherapy for advanced breast cancer. Clin Nucl Med. 1976;1:13-17.

[13]. Janicek MJ, Hayes DF, Kaplan WD. Healing flare in skeletal metastases from breast cancer. Radiology. 1994;192:201-204.

[14]. Johns WD, Garnick MB, Kaplan WD. Leuprolide therapy for prostate cancer:an association with scintigraphic "flare" on bone scan. Clin Nucl Med.1990;15:485-487.

[15]. Lind JS, Postmus PE, Smit EF. Osteoblastic bone lesions developing during treatment with erlotinib indicate major response in patients with non-small cell lung cancer: a brief report.JThorac Oncol. 2010 Apr;5(4):554-7

[16]. Krupitskaya Y, Eslamy HK, Nguyen DD, Kumar A, Wakelee HA.Osteoblastic Bone Flare on F18-FDG PET in Non-small Cell Lung Cancer (NSCLC) Patients Receiving Bevacizumab in Addition to Standard Chemotherapy. J Thorac Oncol. 2009 Mar;4(3):429-31. 
[17]. Armstrong AJ, Kaboteh R, Carducci MA, Damber JE, Stadler WM, Hansen M, et al. Assessment of the bone scan index in a randomized placebo-controlled trial of tasquinimod in men with metastatic castration-resistant prostate cancer (mCRPC). Urol Oncol. 2014;32:1308-16.

[18]. O’Sullivan GJ, Carty FL, Cronin CG. Imaging of bone metastasis: an update. World J Radiol. 2015;7:202-11.

[19]. Bauerle T, Semmler W. Imaging response to systemic therapy for bone metastases. Eur Radiol. 2009;19:2495507.

[20]. Coleman RE, Mashiter G, WhitakerKB, Moss DW, Rubens RD,Fogelman I. Bone scan flare predicts successful systemic therapy for bone metastases. J Nucl Med. 1988;29:1354-9.

[21]. Ortiz A, Lin SH. Osteolytic and osteoblastic bone metastases: two extremes of the same spectrum?Recent Results Cancer Res. 2012;192:225-33.

[22]. Mundy GR.Metastasis to bone: causes, consequences, and therapeutic opportunities. Nat Rev Cancer. 2002 Aug;2(8):584-93.

[23]. Schneider JA, Divgi CR, Scott AM, et al. Flare on bone scintigraphy following Taxol chemotherapy for metastatic breast cancer. J Nucl Med.1994;35:1748 -1752

[24]. Chinese Journal of Nuclear Medicine and molecular imaging, 2018,38(6): 454-454

[25]. Hamaoka T, Madewell JE, Podoloff DA, Hortobagyi GN, Ueno NT. Bone imaging in metastatic breast cancer. J Clin Oncol. 2004 Jul 15;22(14):2942-53.

[26]. Amoroso V, Pittiani F, Grisanti S, Valcamonico F, Simoncini E, Ferrari VD, Marini G. Osteoblastic flare in a patient with advanced gastric cancer after treatment with pemetrexed and oxaliplatin: implications for response assessment with RECIST criteria. BMC Cancer. 2007 Jun 1;7:94.

[27]. MOUNTZIOS G, RAMFIDIS V, TERPOS E, et a1. Prognostic significance of bone markers in patients with lung cancer metastatic to the skeleton: a review of published data[J]. ClinLung Cancer, 2011, 12(6): 341 .

[28]. OYEWUMI MO, ALAZIZI A, WEHRUNG D, et a1. Emerging lung cancer therapeutic targets based on the pathogenesis of bone metastases[J]. Int J Cell Bin1, 2014, 2014: 236.

[29]. Karhade A V, Thio Q C B S, Kuverji M, et al. Prognostic value of serum alkaline phosphatase in spinal metastatic disease[J]. British Journal of Cancer, 2019. 
Table 1. Clinical characteristics of patients

\begin{tabular}{|c|c|c|c|}
\hline Treatment & $\begin{array}{ll}\text { Total } & \text { Case } \\
\text { Number } & \end{array}$ & $\begin{array}{l}\text { Overall Response } \\
\text { (Case Number) }\end{array}$ & $\begin{array}{l}\text { Number of Bone } \\
\text { Flares }\end{array}$ \\
\hline Bevacizumab $+\mathrm{TC}$ & 35 & $\begin{array}{l}\text { PR (17) } \\
\text { SD (15) } \\
\text { PD (3) }\end{array}$ & $\begin{array}{l}2 \\
2\end{array}$ \\
\hline $\mathrm{PD}-1+\mathrm{PC}$ & 5 & $\begin{array}{l}\text { PR (1) } \\
\text { SD (1) } \\
\text { PD (3) }\end{array}$ & 1 \\
\hline $\mathrm{PC}$ & 6 & $\begin{array}{l}\text { PR (2) } \\
\text { SD (2) } \\
\text { PD (1) } \\
\text { CR (1) }\end{array}$ & \\
\hline EGFR-TK1 & 11 & $\begin{array}{l}\text { PR (4) } \\
\text { SD (5) } \\
\text { PD (2) }\end{array}$ & $\begin{array}{l}1 \\
1\end{array}$ \\
\hline Total & 57 & $\begin{array}{l}\text { PR (24) } \\
\text { SD (23) } \\
\text { PD (9) } \\
\text { CR (1) }\end{array}$ & $\begin{array}{l}7 \\
4 \\
3\end{array}$ \\
\hline
\end{tabular}

TC: taxol +carbo; PC: pemetrexed disodium + carbo; PR: partial response; SD: stable disease; PD:progressive disease; CR:complete response 
Table 2. Clinical characteristics of patients with flare phenomenon

\begin{tabular}{|c|c|c|c|c|c|c|c|}
\hline Patients & Treatment & $\begin{array}{l}\text { Bone } \\
\text { Metastases } \\
\text { at Baseline }\end{array}$ & $\begin{array}{l}\text { Time of } \\
\text { Treatment } \\
\text { beginning }\end{array}$ & $\begin{array}{l}\text { Overall } \\
\text { Response }\end{array}$ & $\begin{array}{l}\text { Time of } \\
\text { progressive } \\
\text { disease }\end{array}$ & $\begin{array}{l}\text { Time of Bone } \\
\text { Flare }\end{array}$ & $\begin{array}{l}\text { Days of } \\
\text { Occurrence } \\
\text { of Bone } \\
\text { Flare }\end{array}$ \\
\hline 1 & PD-1+PC & Yes & 2019.02 .22 & PR & - & 2019.4 .4 & 45 \\
\hline 2 & $\begin{array}{l}\text { Bevacizuma } \\
\mathrm{b}+\mathrm{TC}\end{array}$ & Yes & 2018.6.11 & SD & 2018.12 .7 & 2019.10 .8 & 120 \\
\hline 3 & EGFR-TK1 & Yes & 2019.1 .9 & SD & - & 2019.2.21 & 45 \\
\hline 4 & EGFR-TK1 & Yes & 2019.1 .8 & PR & - & 2019.2.18 & 42 \\
\hline 5 & $\begin{array}{l}\text { Bevacizuma } \\
\mathrm{b}+\mathrm{TC}\end{array}$ & Yes & 2018.10 .22 & PR & 2019.04 & 2018.11 .26 & 41 \\
\hline 6 & $\begin{array}{l}\text { Bevacizuma } \\
\mathrm{b}+\mathrm{TC}\end{array}$ & Yes & 2018.3.12 & PR & 2018.12 .27 & 2018.4.24 & 43 \\
\hline 7 & $\begin{array}{l}\text { Bevacizuma } \\
\mathrm{b}+\mathrm{TC}\end{array}$ & Yes & 2018.4.10 & $\mathrm{SD}$ & 2019.2.19 & 2018.8 .13 & 125 \\
\hline
\end{tabular}


Figures
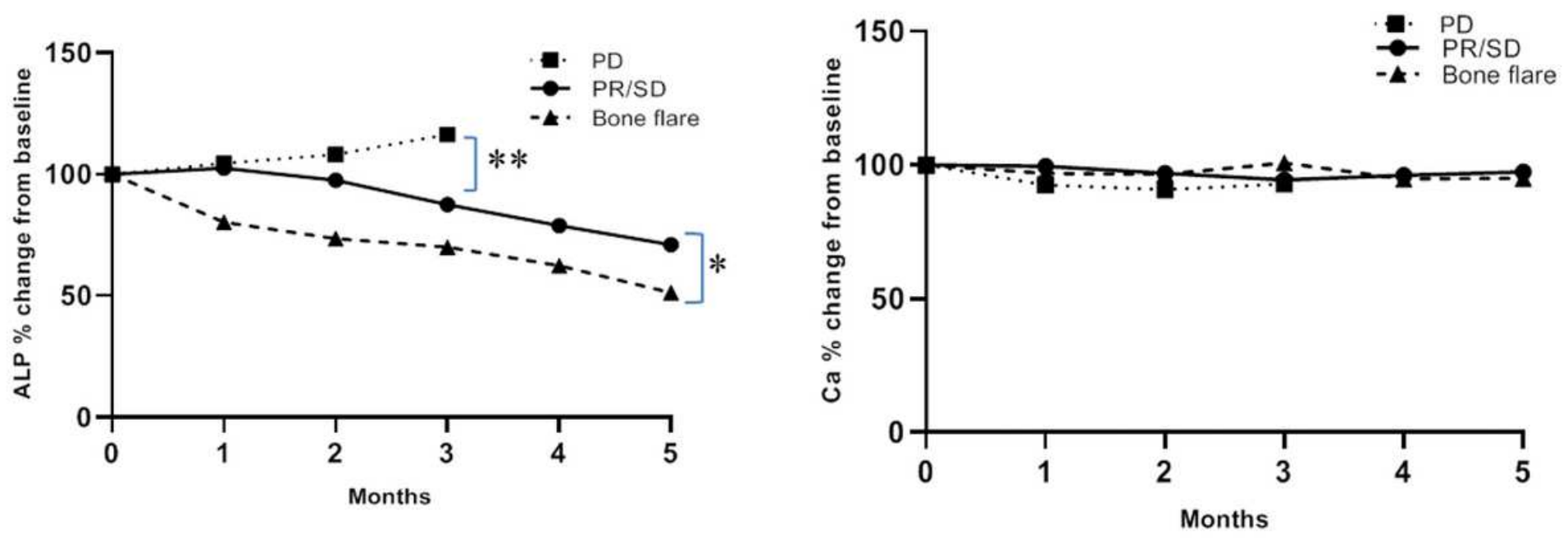

Figure 1

Significant change in serum ALP level but not $\mathrm{Ca} 2+$ upon patients displayed bone flare (A-B). Serum ALP (A) and $\mathrm{Ca} 2+(B)$ during systemic therapy in 25 patients. Data presented as $\%$ change from baseline for each group. Points represent median values. Baseline $=100 \% .{ }^{*} P<0.05 * * P<0.01$, 

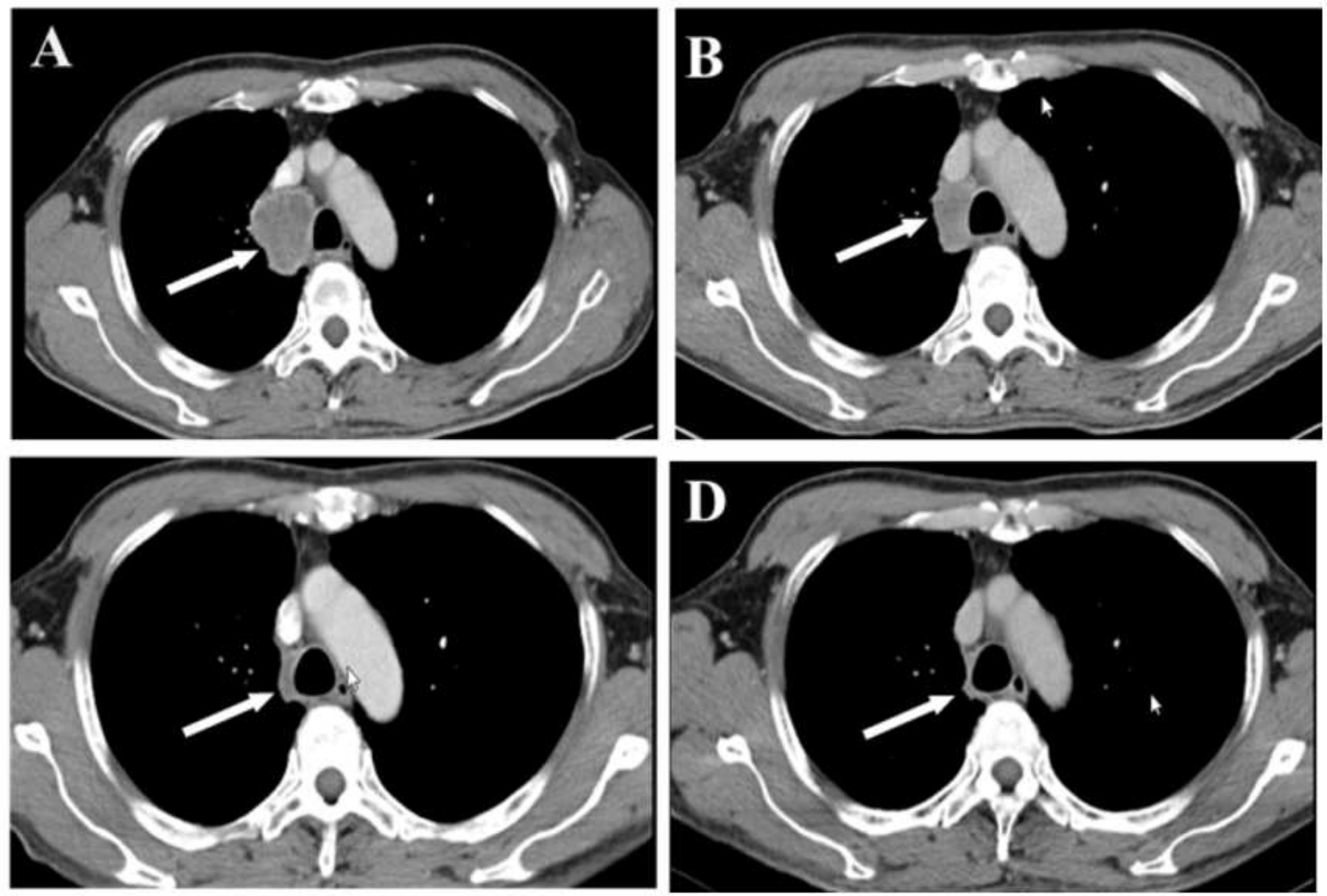

Figure 2

Changes of mediastinal lymph nodes before and after therapy in \#1 patient of typical bone flare. (A).The baseline study of chest CT images shows lymphadenopathy in 4R region of mediastinum, with $3.5 \mathrm{~cm}$ short diameter and enhanced in margins. (B).After 2-cycle treatment, the chest CT images shows lymphadenopathy in the $4 \mathrm{R}$ region of the mediastinum with a short diameter of $2.2 \mathrm{~cm}$. (C). After 4-cycle treatment, the chest CT images shows lymphadenopathy in 4R region of mediastinum with a short diameter $1.0 \mathrm{~cm}$. (D). After 6-cycle treatment, the chest CT images shows that the lymphadenopathy of the mediastinum in $4 \mathrm{R}$ region with short diameter $0.5 \mathrm{~cm}$ 

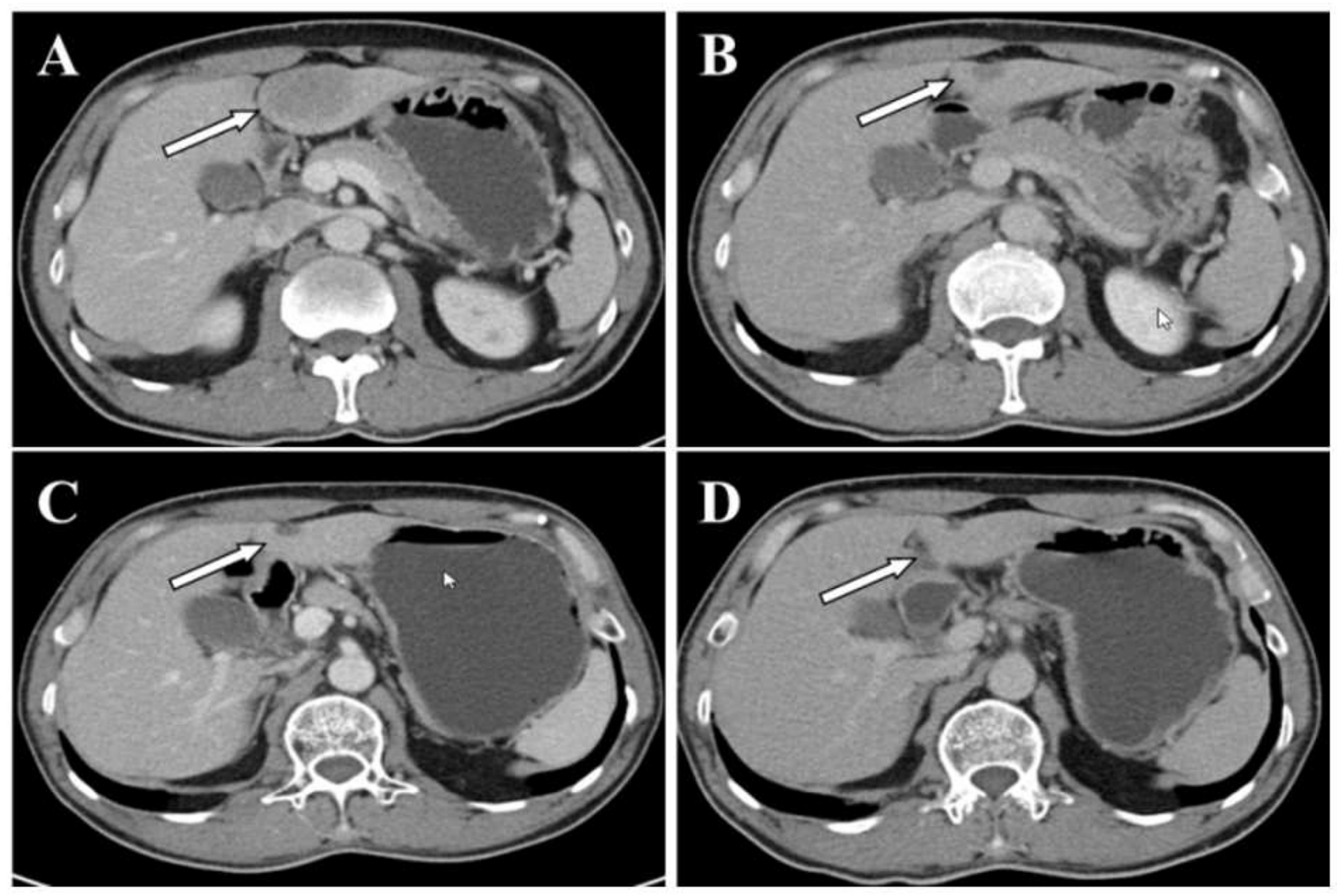

\section{Figure 3}

Changes of liver metastases before and after treatment in \#1 patient with typical bone flare. (A).The baseline abdominal CT images show a lump shadow, $3.2 \mathrm{~cm} \times 4.6 \mathrm{~cm}$, in the left lateral lobe of the liver. The edge enhancement of the lesion was obvious after enhanced CT scanning, and the enhancement of the lesion after delay was lower than that of the surrounding liver tissue. (B). After 2-cycle treatment, abdominal CT images show a lump shadow of $0.8 \mathrm{~cm} \times 1 \mathrm{~cm}$ in the left lateral lobe of the liver. (C). After 4-cycle treatment, abdominal CT showed a mass in the left lateral lobe of the liver with a long diameter of about $10 \mathrm{~mm}$. (D).After 6-cycle treatment, the abdominal CT showed a lump shadow in the left lateral lobe of the liver with a long diameter of about $7 \mathrm{~mm}$. Arrow shows the lump shadow. 

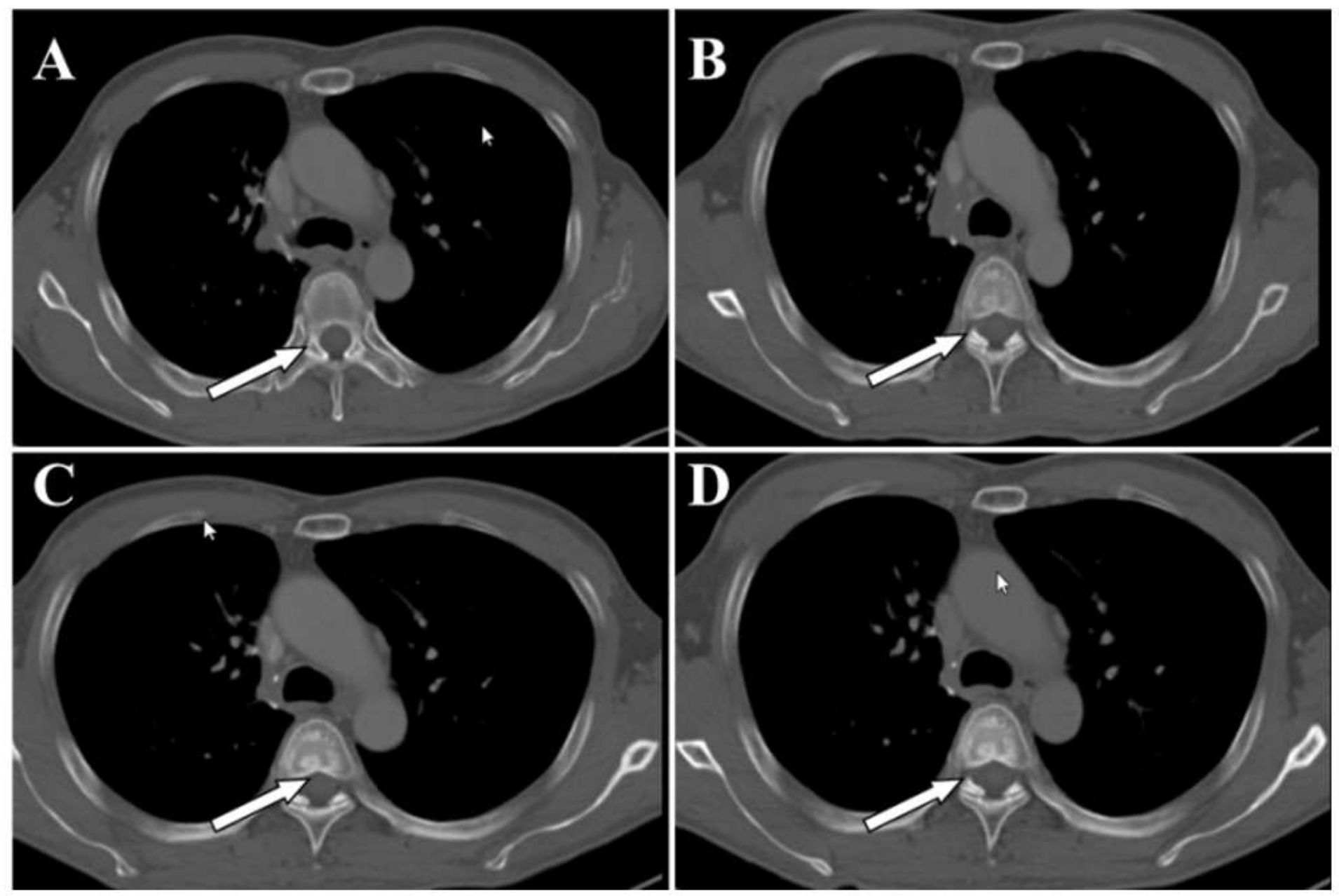

\section{Figure 4}

The changes of bone destruction of Thoracic Vertebra 5 in \#1 patient with typical bone flare after treatment. (A). No obvious bone abnormalities were found in the T5 vertebrae before treatment. (B). After 2-cycle treatment, the bone destruction of T5 vertebral body was found and bone metastasis was developped. (C). After 4-cycle treatment, bone destruction of the T5 vertebral body was similar to that of before treatment. (D). After 6-cycle treatment, bone destruction of the T5 vertebral body was similar to that of before treatment. 

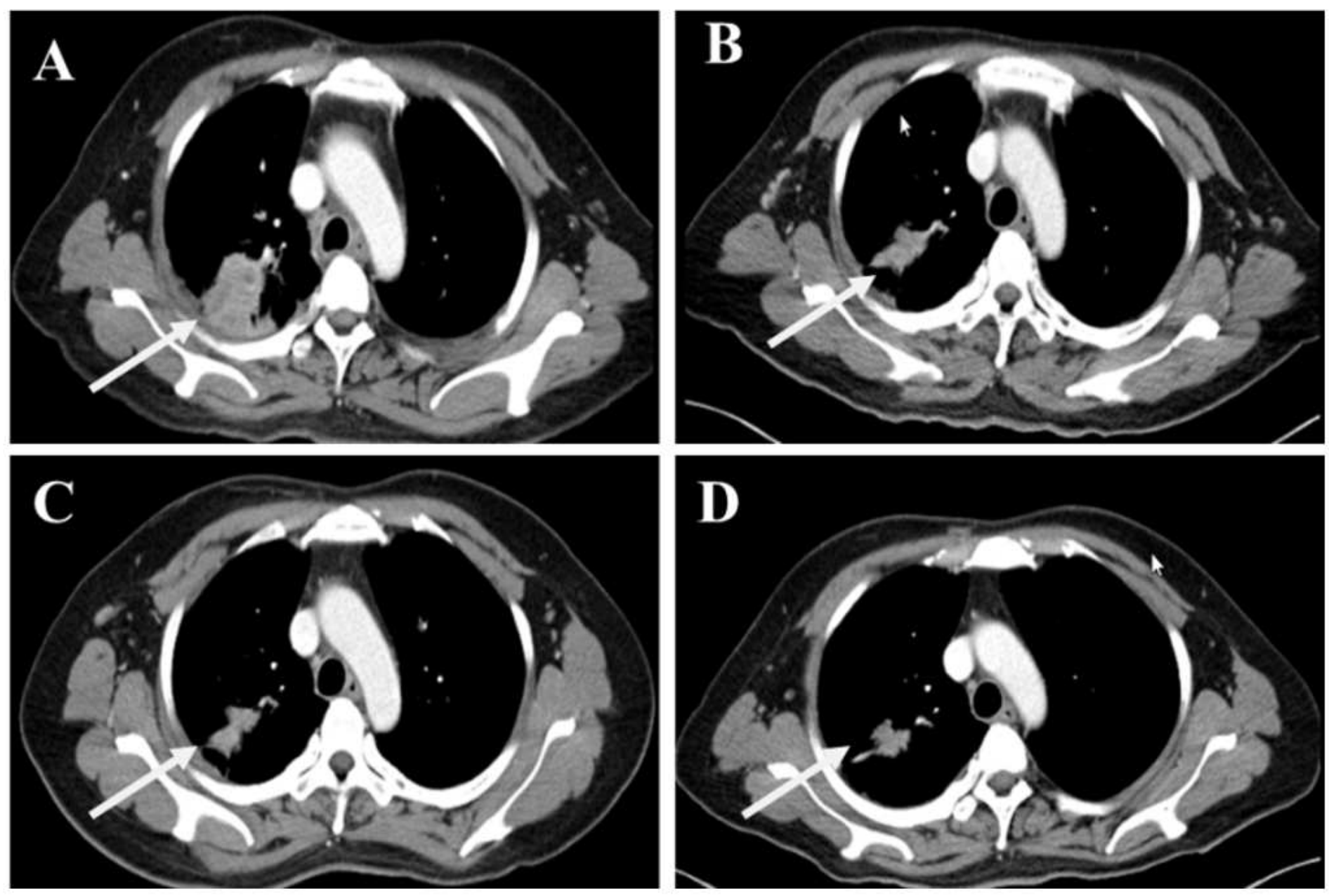

\section{Figure 5}

Changes of pulmonary lesions before and after treatment in \#2 patient with typical bone flare. (A). At baseline, chest CT image showed that the soft tissue mass in the right upper lobe of the lung was irregular and inhomogeneous with a size of $2.9 \mathrm{~cm} \times 3.8 \mathrm{~cm}$ and displaying rough margin and thickening of adjacent Pleura. (B). After 2 cycles of treatment, chest CT showed a right upper lobe soft tissue mass with irregular and heterogeneous enhancement with a size about $2.1 \mathrm{~cm} \times 3.8 \mathrm{~cm}$ and rough margin. (C). After 4-cycle treatment, chest CT image showed a right upper lobe soft tissue mass with irregular, heterogeneous enhancement, about $2.1 \mathrm{~cm} \times 3.5 \mathrm{~cm}$ with rough margin and thickening of adjacent Pleura. (D). After 6-cycle of treatment, chest CT showed a right upper lobe soft tissue mass with irregular, heterogeneous enhancement, about $2.0 \mathrm{~cm} \times 3.5 \mathrm{~cm}$ with rough margin and thickening of adjacent pleura. 

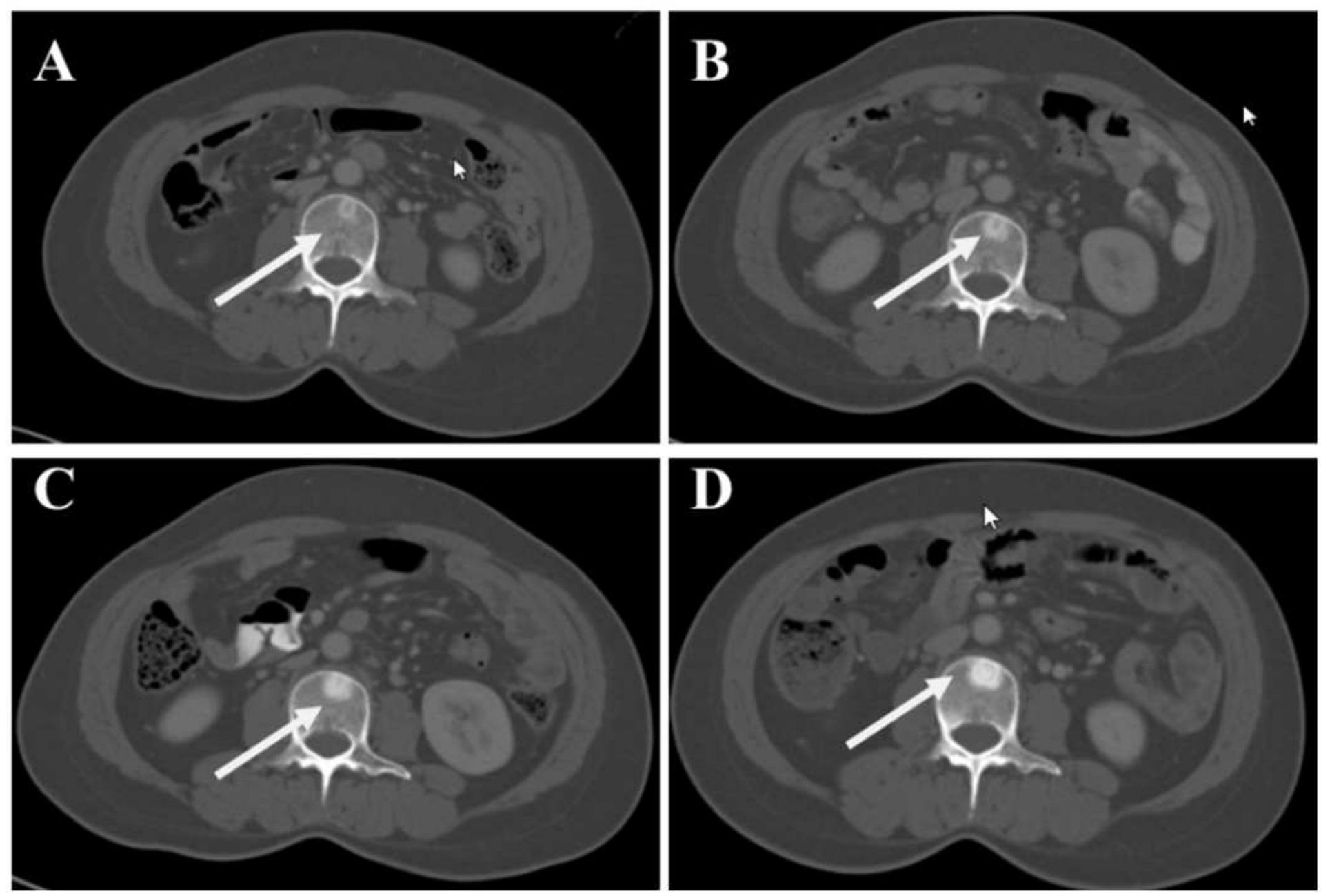

Figure 6

Changes of bone metastases lesions in \#2 patient with typical bone flare before and after the treatment. (A). At baseline PELVIC CT images showed bony destruction and soft tissue formation in the lumbar spine, which was dignosed bone metastases. (B). After 6 weeks of targeted therapy, PELVIC CT showed bone destruction and soft tissue formation in lumbar vertebrae, and bone metastasis, which was larger than before.(C). After 12-week targeted therapy, PELVIC CT showed bone destruction and soft tissue formation of lumbar vertebrae, which was larger than before. (D). After 18-week targeted therapy, PELVIC CT showed bone destruction and soft tissue formation of lumbar vertebrae, which was larger than before 


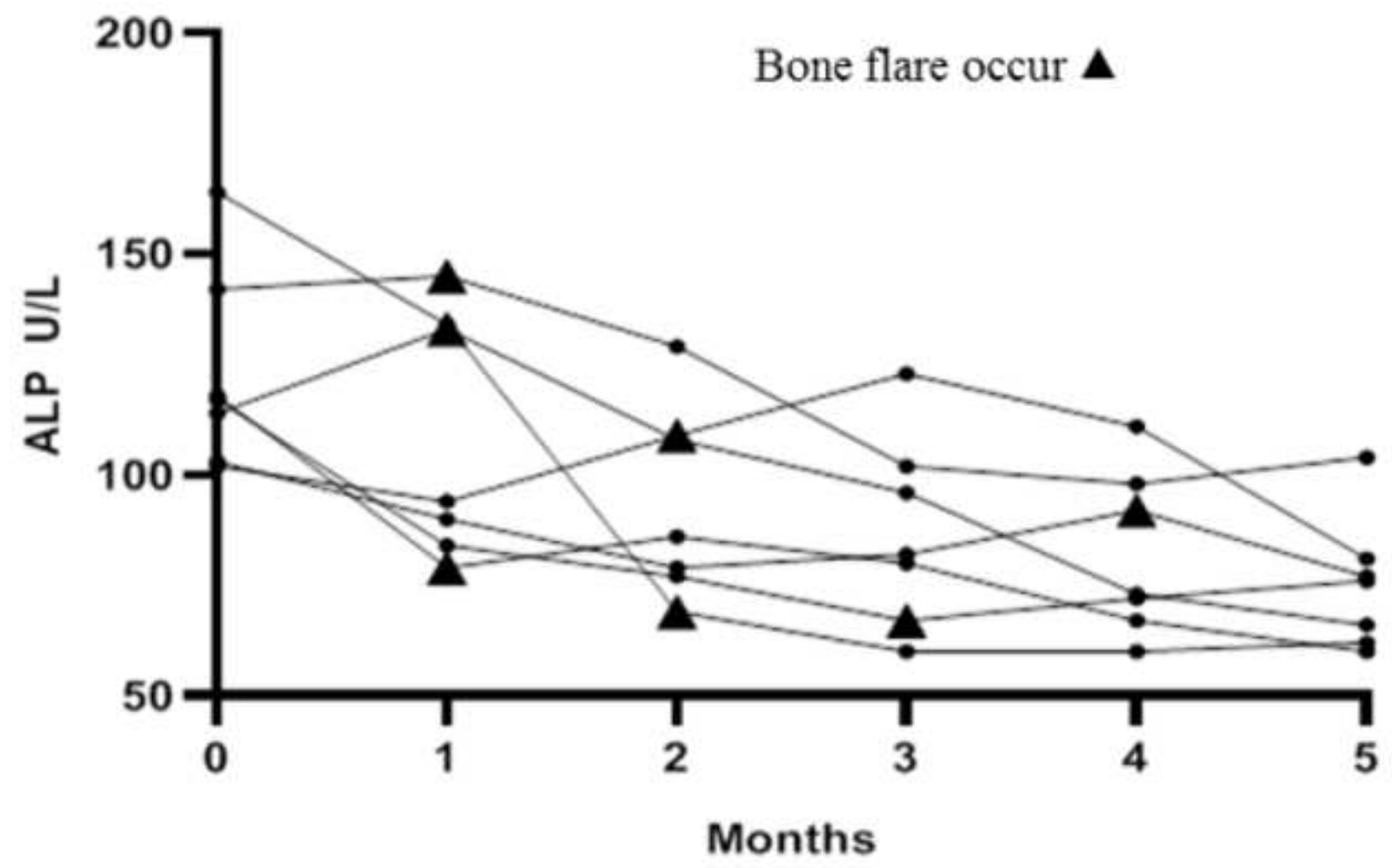

Figure 7

Serial changes in serum ALP changes before and after treatment in 7 patients with bone flare The triangular symbols in this figure show the time and ALP levels of bone flare found in 7 patients during treatment. 